\title{
Properties of dust and PAHs in various environments of nearby galaxies
}

\author{
Hidehiro Kaneda* \\ Graduate School of Science, Nagoya University, Nagoya 464-8602, Japan \\ E-mail: kaneda@u.phys.nagoya-u.ac.jp
}

In star-forming regions, dust and polycyclic aromatic hydrocarbons (PAHs) absorb a significant fraction of stellar ultraviolet (UV) photons and re-radiate them in the infrared (IR). Hence the IR luminosities due to dust and PAH emission are both powerful tools to trace star-forming activities in galaxies. However they are not merely tracers of star-formation activity. Spectral information on the dust and PAH emission would have much deeper physical implications for understanding the properties of the ISM. External galaxies provide us with a much wider range of the ISM physical conditions than our Galaxy. For example, Spitzer mid-IR spectroscopy has shown a variety of the PAH emission features for various types of galaxies, which reflects significant changes in the properties of PAHs, such as an ionization state and a size distribution. AKARI near-IR spectroscopy has revealed spatial variations in the intensity ratio of the aromatic to the aliphatic hydrocarbon spectral feature in galaxies, indicating structural changes of carbonaceous grains in shocked regions.

The relation of PAH to far-IR dust is another important probe to study the conditions of the interstellar environments. In general, PAHs and far-IR dust are mixed well in the ISM, producing global correlations between their IR luminosities. In local areas within a galaxy, however, their abundance ratios are expected to vary substantially due to interactions with interstellar shocks, hard UV radiation fields and diffuse X-ray hot plasmas. Until recently, spatial resolutions in the far-IR were extremely poor compared with those in the near- and mid-IR. Herschel now enables us to make a detailed comparison of the spatial distributions of far-IR dust and PAH emission within a galaxy.

This paper reviews recent observational results on the IR emission properties of dust and PAHs exposed to various environments of nearby galaxies, which range from passive elliptical galaxies to starburst galaxies with galactic superwinds, and (ultra-)luminous infrared galaxies. In particular, the paper focuses on variations in the PAH spectral features and PAH to far-IR dust ratios to discuss their implications for the processing of carbonaceous grains in harsh interstellar environments to understand the fate of dust processed by shocks and radiation through the evolution of galaxies.

The Life Cycle of Dust in the Universe: Observations, Theory, and Laboratory Experiments 18-22 November, 2013

Taipei, Taiwan

*Speaker. 


\section{Infrared observations of dust in nearby galaxies}

Nearby galaxies provide a wide range of the physical and chemical conditions of the interstellar medium (ISM), compared to our Galaxy, the Large Magellanic Cloud (LMC) and the Small Magellanic Cloud (SMC). These ISM conditions include the intensity and hardness of interstellar radiation field, metallicity, stellar population, large-scale shocks, hot plasma environments, and so on. Their proximity ( $\lesssim 10 \mathrm{Mpc}$ ) enables us to perform spatially-resolved studies on the ISM structures of galaxies, although they are not close enough to study interaction between stars and the ISM. The latter study can be carried out for our Galaxy and the LMC/SMC, and therefore nearby galaxies are complementary to Galactic and Magellanic targets for the ISM studies. Faint galaxies, such as dwarf and elliptical galaxies, can only be studied when they are relatively nearby $(\lesssim$ $10 \mathrm{Mpc}$ ). Accurate calibration of infrared (IR) dust emission in nearby galaxies as star-formation indicators is also important for study of distant galaxies.

Space IR observations of dust in nearby galaxies started with IRAS in 1983; IRAS detected more than 25000 galaxies, which included spiral galaxies, Seyfert galaxies and even early-type galaxies [1, 2, 6, 4]. IRAS discovered new classes of IR-bright galaxies such as luminous IR galaxies (LIRGs) and ultraluminous IR galaxies (ULIRGs) [5, 6]. Following the photometric surveys by IRAS, ISO performed IR spectroscopic observations of nearby galaxies, which covered a wavelength range from 2 to $200 \mu \mathrm{m}$ continuously [7, 8]. In particular, spectroscopic studies of polycyclic aromatic hydrocarbons (PAHs) for galaxies in the near- and mid-IR were initiated with ISO, which were carried out not only for IR-bright galaxies but also for normal galaxies [9].

Following IRAS and ISO, Spitzer enabled us to study nearby galaxies with high sensitivities and high imaging capabilities especially in the mid-IR, thanks to the improvement in the detector technologies. AKARI performed all-sky surveys in the mid- and far-IR with higher sensitivities and spatial resolutions than IRAS. Recently Herschel realized unprecedentedly high spatial resolutions in the far-IR thanks to a 3.5-m-diameter telescope, which detected detailed structures of the cold ISM in nearby galaxies. Spectroscopically, high-sensitivity 5-35 $\mu \mathrm{m}$ and $2.5-5 \mu \mathrm{m}$ spectra of nearby galaxies were obtained with Spitzer/IRS and AKARI/IRC, respectively. A sub-mm wavelength coverage beyond $200 \mu \mathrm{m}$ was realized with Herschel/SPIRE. There are many observational programs which systematically study nearby galaxies with Spitzer, AKARI, or Herschel, such as SINGS [10], ISMGN [11], KINGFISH [12], and DGS [13].

With Spitzer/IRS, detailed studies of the PAH emission features are carried out based on the mid-IR spectra of the SINGS sample [14]. It is found that the PAH features are notably similar among star-forming galaxies. Spitzer/IRS improves the determination of the shapes and the overall strengths of the PAH profiles. One of the Spitzer/IRS findings is the $17 \mu \mathrm{m}$ broad feature commonly detected in galaxies, which shows a very tight correlation with the $11.3 \mu \mathrm{m}$ feature [14]. The feature is likely due to relatively large PAHs in a C-C-C bending mode. More recently, significant variations of the PAH spectral features were discovered from galaxy to galaxy; early-type galaxies show unusually weak 6-9 $\mu \mathrm{m}$ PAH features relative to the features at longer wavelengths (Fig.1) [15, 16, 17, 18]. According to recent results, about half of the Spitzer/IRS sample of 91 nearby early-type galaxies show the PAH emission, and 80-90\% of them show similarly weak 6-9 $\mu \mathrm{m}$ PAH features [19, 20]. The low ratios of the $7.7 \mu \mathrm{m}$ to the $11.3 \mu \mathrm{m}$ feature can be explained by predominance of either large PAHs or neutral PAHs. The low ratios can be more efficiently 

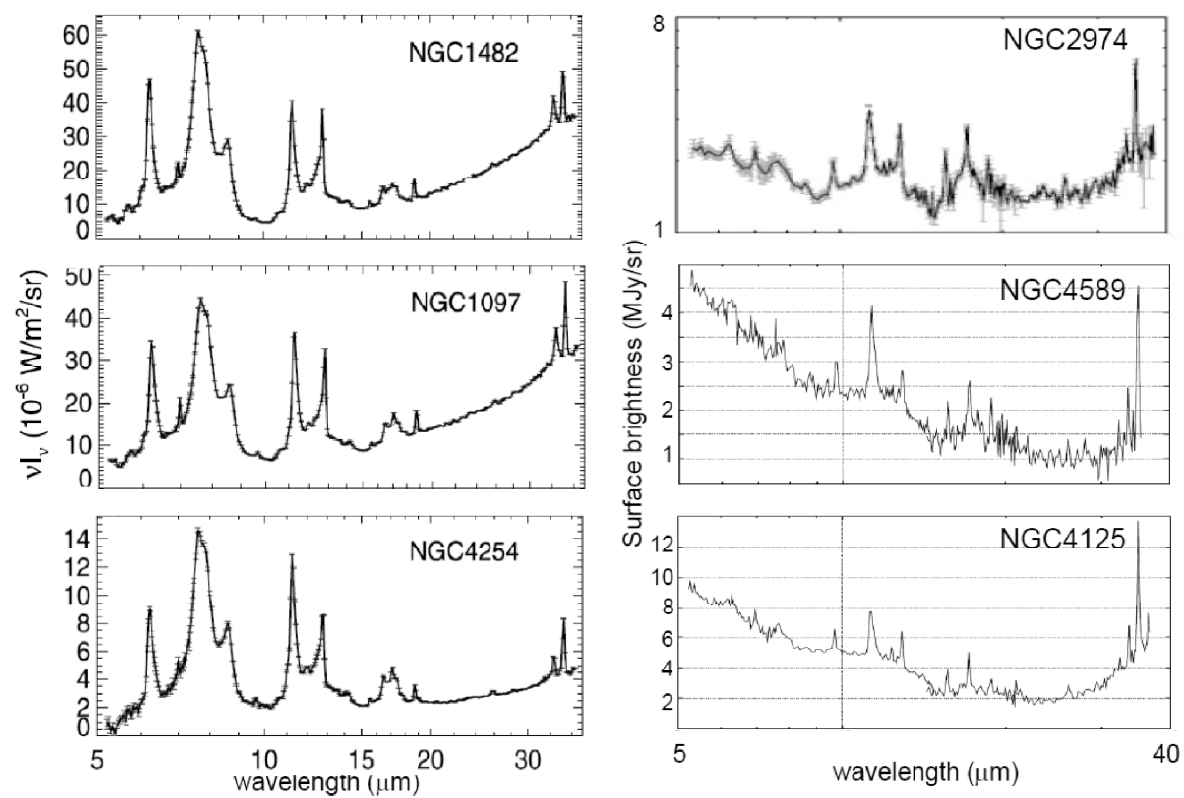

Figure 1: Mid-IR spectra of nearby galaxies obtained with Spitzer/IRS: (left) star-forming galaxies [14 and (right) elliptial galaxies [18]. Note that strong PAH features at 6-9 $\mu \mathrm{m}$ in the left panel are faint in the right panel.

reproduced by predominance of neutral PAHs [21], the situation of which is more reasonable for soft radiation field due to old stars in elliptical galaxies [17, 18].

The correlations between the spatial distributions of PAHs, hot dust and cold dust in nearby galaxies were studied using Spitzer/IRAC and MIPS imaging data. Bendo et al. [22] clearly showed that the IRAC $8 \mu \mathrm{m} /$ MIPS $24 \mu \mathrm{m}$ band intensity ratios are low in HII regions, while PAHs (IRAC $8 \mu \mathrm{m}$ ) are well correlated with cold dust (MIPS $160 \mu \mathrm{m}$ ). They interpreted that PAHs are selectively destroyed due to intense radiation in HII regions. The metallicity effects on the PAH emission and IR colors were also revealed by Spitzer. It was found that a threshold for the presence of PAHs exists around $12+\log (\mathrm{O} / \mathrm{H})=8[23]$ and also that the dust temperature increases with decreasing metallicity [24].

Herschel/PACS and SPIRE enable us to map the properties of cold dust in nearby galaxies. Using the KINGFISH sample, Galametz et al. [25] derived the spatial distributions of dust temperature $T$ and emissivity power-law index $\beta$ in galaxies to find an anti-correlation between $\beta$ and $T$. Rémy-Ruyer et al. [26] systematically investigated the properties of cold dust in low-metallicity dwarf galaxies, combining 48 DGS galaxies with $12+\log (\mathrm{O} / \mathrm{H})=7.0-8.5$ and $61 \mathrm{KINGFISH} \mathrm{galax-}$ ies with $12+\log (\mathrm{O} / \mathrm{H})=7.8-8.7$. They found a systematic change in the $70-500 \mu \mathrm{m}$ spectral energy distributions (SEDs) with metallicity; dust in the DGS sample is warmer than in the KINGFISH sample, but there is no systematic difference in $\beta$ between the samples. They also found excess emission at $500 \mu \mathrm{m}$ on top of the modified blackbody dust continuum from a substantial fraction of their sample galaxies. This may be due to very cold $(\sim 10 \mathrm{~K})$ dust, which calls for unrealistically high dust-to-gas mass ratios. Another possibility is spinning dust, the origin of which is thought to be PAHs. This scenario is, however, inconsistent with the observational trend that low-metallicity 
dwarf galaxies show relatively low PAH abundances. The excess may be related to the nature of amorphous solid opacity [27]. Very recently, metallic particles were also proposed for the origin of the $500 \mu \mathrm{m}$ excess [28]. Although the excess emission has potentially important physical implications, the presence of the excess itself is still controversial; Kirkpatrick et al. [29] reported no substantial excess at $500 \mu \mathrm{m}$ for the KINGFISH sample, which may be related to metallicity effects.

\section{Interpretations based on dust SED models}

Proper modeling of dust SEDs is indispensable to physically interpret IR observational data of nearby galaxies. In the classical dust model established by Désert, Boulanger, and Puget [30], three dust components are incorporated, namely PAHs, carbonaceous very small grains (VSGs) amd big grains (BGs). The dust size distribution is chosen to reproduce the observed extinction (and emission). Since then, every dust model, in principle, assumed these components similarly, but there is a significant difference in the grain opacity from model to model; for example, the Draine \& Li model (DL07) [21] adopted the optical properties of graphite [31], while the DustEM model [32] used those of hydrogenated amorphous carbon (HAC) [33] for carbonaceous dust. The radiation field intensity is usually given in units of the starlight intensity in the solar neighborhood, $U$, which is defined in a photon energy range of $0-13.6 \mathrm{eV}$ [34]. This is compared to the far-UV $(6-13.6 \mathrm{eV})$ interstellar radiation field, $G_{0}$, used in the PDR model. For galaxies, the mass of dust $M_{\text {dust }}(U)$ illuminated by the radiation field $U$ is assumed to follow a power-law distribution $d M_{\text {dust }}(U) \propto U^{-\alpha} d U$ [35]. Before the Spitzer era, Galliano et al. [36] created a sophisticated dust SED model, in which they determined the interstellar radiation field self-consistently through UV-optical and IR-mm observations with IR ionic lines. Applying the model to low-metallicity galaxies, they found the presence of very cold dust (5-7 K) occupying almost a half of the total dust mass [37]. They pointed out that most grains are likely to be in a stochastic heating mode in the low-metallicity environments [37.

In the Spitzer and Herschel eras, one of the most popular dust SED models for studies of nearby galaxies has been the DL07 model [21], in which the treatment of the PAH component has been improved much, compared to the previous models. In the model, the interstellar radiation field, to which dust is exposed, consists of two components: the general diffuse ISM component represented by a single radiation field strength $U_{\min }$ and photodissociation region (PDR) component which has a power-law distribution from $U_{\min }$ to $U_{\max }$. By applying the dust model to the SEDs of the Spitzer/SINGS sample, it was found that the fractional mass of PAHs to the total dust, $q_{\mathrm{PAH}}$, shows a significant variation from galaxy to galaxy with a median value of $3.5 \%$ [38]. With the model, it was clearly shown that dust in the diffuse ISM dominates the IR power (70-90\%) rather than dust in PDRs. Even when Herschel data points are added to the Spitzer SEDs for the KINGFISH sample, the model parameters are found to be relatively unchanged, such as $q_{\mathrm{PAH}}, U_{\mathrm{min}}$, $M_{\text {dust }}$. except that the fraction of dust heating attributed to intense radiation fields associated with PDRs is found to be $\sim 20 \%$ larger when Herschel data are included in the analysis [39].

The high spatial resolution of Herschel imaging data enables to perform spatially-resolved SED fitting to a combination of the Spitzer mid-IR and Herschel far-IR data. Using the DL07 model, dust-to-gas mass ratio maps were created for NGC 628 and NGC 6946 [40]. As a result, the 


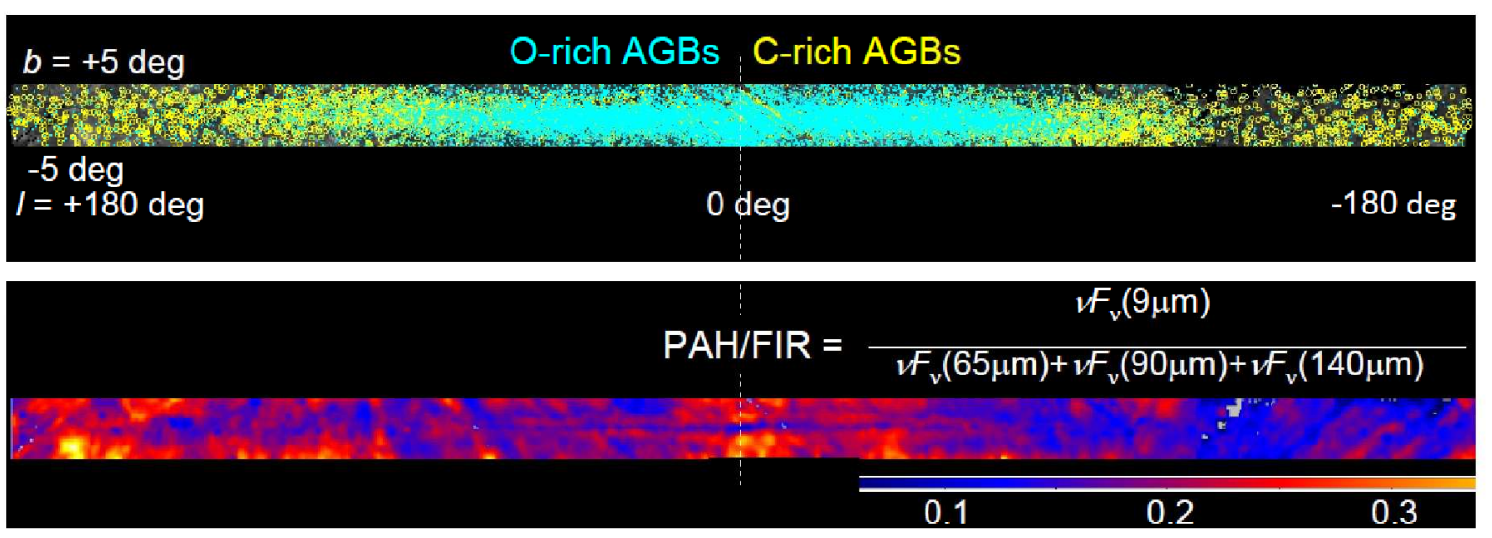

Figure 2: (Top) Spatial distribution of oxygen-rich and carbon-rich AGB stars along the Galactic plane [47]. (Bottom) Distribution of PAH/far-IR intensity ratios, as defined by the equation in the panel, along the Galactic plane. Both distributions are obtained using the AKARI all-sky survey data.

dust-to-gas mass ratio is found to vary by almost one order of magnitude $(\sim 0.004-0.04)$ within the galaxies [40]. The high spatial resolution of Herschel data also allows for studying the dependence of the PAH abundance on the metallicity for individual HII regions in galaxies [41]; the result suggested that the dependence is not caused by metallicity-dependent formation of PAHs, but rather by their destruction; a similar result was reported for the low-metallicity environ ment in the SMC [42].

The DustEM model adopts amorphous carbon instead of graphite which is used in the DL07 model. The change of amorphous carbon from graphite results in lowering the dust mass derived by the model fitting by a factor of $2-3$, because of the higher far-IR to sub-mm emissivity for amorphous carbon [43]. Observationally $80-100 \%$ of the $\mathrm{C}$ atoms in shocked regions are in the gas phase [44], while 30-100 \% of dust is expected to be destroyed by $\sim 100 \mathrm{~km} / \mathrm{s}$ shocks for amorphous carbon, but only $15 \%$ for graphite [45, 46]. Therefore the assumption of amorphous carbon for carbonaceous grains might be better to explain the gas-phase abundance of $\mathrm{C}$ atoms observed in shocked regions.

A dominant dust population may be different between far-IR and sub-mm regions due to higher absorption efficiency of amorphous carbon in the near-IR than amorphous silicate, causing carbonaceous dust to have higher temperatures than silicate dust [32, 21]. Figure 2 shows the spatial distributions of carbon-rich (C-rich) and oxygen-rich (O-rich) asymptotic giant branch (AGB) stars, derived using the color-color diagrams of the 9 and $18 \mu \mathrm{m}$ band fluxes in the AKARI all-sky pointsource catalog, with the 2MASS $J, H$, and $K$ band fluxes [47]. As can be seen in the figure, the O-rich AGBs are more concentrated toward the Galactic center, while the C-rich AGBs are rather uniformly distributed throughout the Galactic plane. The lower panel indicates that interstellar PAHs and far-IR dust grains are similar in the spatial distribution, in contrast to the difference in the distribution between the dominant suppliers of carbonaceous and silicate grains, C-rich and O-rich AGBs. This may suggest that silicate grains do not make a significant contribution to the far-IR emission in the diffuse ISM, but to the sub-mm regions.

As to the behavior of the sub-mm opacity of amorphous grains, pure solid-state physics can 
explain the change of the emissivity power-law index $\beta$ with physical parameters [27]. One component to be considered for the sub-mm opacity is related to the disordered charge distribution with the correlation length for charge neutrality [48], which predicts an increase of $\beta$ up to 4 with wavelength. Another is expressed as two level systems causing charge transition by tunneling effects due to defects in lattice, which predicts a decrease of $\beta$ with temperature [49]. Thus the sub-mm opacity depends on the physical properties of amorphous grains, especially the degree of processing, but not chemical composition much. Although the origin of the $500 \mu \mathrm{m}$ excess indicated by the Herschel data is still quite uncertain, the sub-mm opacity potentially delivers important information on the nature of grains, and its proper treatment should be required for future dust SED models.

\section{Properties of dust in active galaxies}

This section and the next describe several results on the properties of dust observed for particular targets of active and passive galaxies, respectively. Among the former galaxies, nearby edge-on starburst galaxies with prominent galactic superwinds are important targets to understand the processing of dust through material circulation on a galactic scale. For M 82, for example, Spitzer, AKARI, and Herschel detect copious amounts of BGs and PAHs flowing out of the disk through galactic superwinds [50, 51, 52]. In total, $\sim 25 \%$ of all the dust has been expelled from the disk [52, 53]. Figure 3 shows the distribution of the PAH emission in M 82, which is widely extended toward the halo regions. It is found that there is an excellent correlation between the PAH and $\mathrm{H} \alpha$ distributions [51]. The spectro-polarimetry showed that $\mathrm{H} \alpha$ is significantly $(5-15 \%)$ polarized [54], which suggests that $\mathrm{H} \alpha$ photons from the galactic disk are scattered by dust grains in the halo toward us. The excellent spatial correlation can be explained such that the PAHs are produced by shattering of the dust grains by shocks [55]. Moreover it was found that the dust flow velocity estimated from a shift of the $\mathrm{H} \alpha$ line decreases from $\sim 200 \mathrm{~km} \mathrm{~s}^{-1}$ down to $\sim 0 \mathrm{~km} \mathrm{~s}^{-1}$ with the height from the disk $(0-1.4 \mathrm{kpc})$, which implies that the dust grains thus processed are likely to be falling back toward the disk [54].

As also shown in Fig. 3, using the AKARI near-IR spectroscopy, the PAH $3.3 \mu$ m emission and the 3.4-3.6 $\mu \mathrm{m}$ features are clearly detected in the halo regions, which are located at a distance of $2 \mathrm{kpc}$ away from the galactic center, thus confirming the presence of PAHs even in the harsh environment of the M82 halo [56]. The observed spectral properties are quite different from those commonly seen in other spiral galaxies; the 3.4-3.6 $\mu \mathrm{m}$ features are unusually strong in the halo, suggesting the dominance of aliphatic structures over aromatic ones. From the 34 near-IR spectra taken from the center, disk and halo regions of M82, it is revealed that the aliphatic to aromatic ratios systematically increase with the distance from the center, which seems to be consistent with the above picture of shattering of carbonaceous grains by shocks in the halo.

For about 200 star-forming galaxies in the redshift range of $\sim 0.01-0.1$, a global relation is investigated between the PAH $3.3 \mu \mathrm{m}$ luminosity, $L_{3.3}$, and the total IR $(8-1000 \mu \mathrm{m})$ luminosity, $L_{\text {IR }}$ [57]. The sample is classified into IR galaxies (IRGs; $L_{\text {IR }}<10^{11} L_{\odot}$ ), luminous IR galaxies (LIRGs: $L_{\mathrm{IR}} \sim 10^{11}-10^{12} L_{\odot}$ ) and ultra-luminous IR galaxies (ULIRGs: $L_{\mathrm{IR}}>10^{12} L_{\odot}$ ). It is confirmed that many of the IRGs and LIRGs follow the relationship $L_{3.3} / L_{\mathrm{IR}} \simeq 10^{-3}$, which is a ratio typical of starburst galaxies [58]. At the same time, it is also found that the $L_{3.3} / L_{\mathrm{IR}}$ ratio 


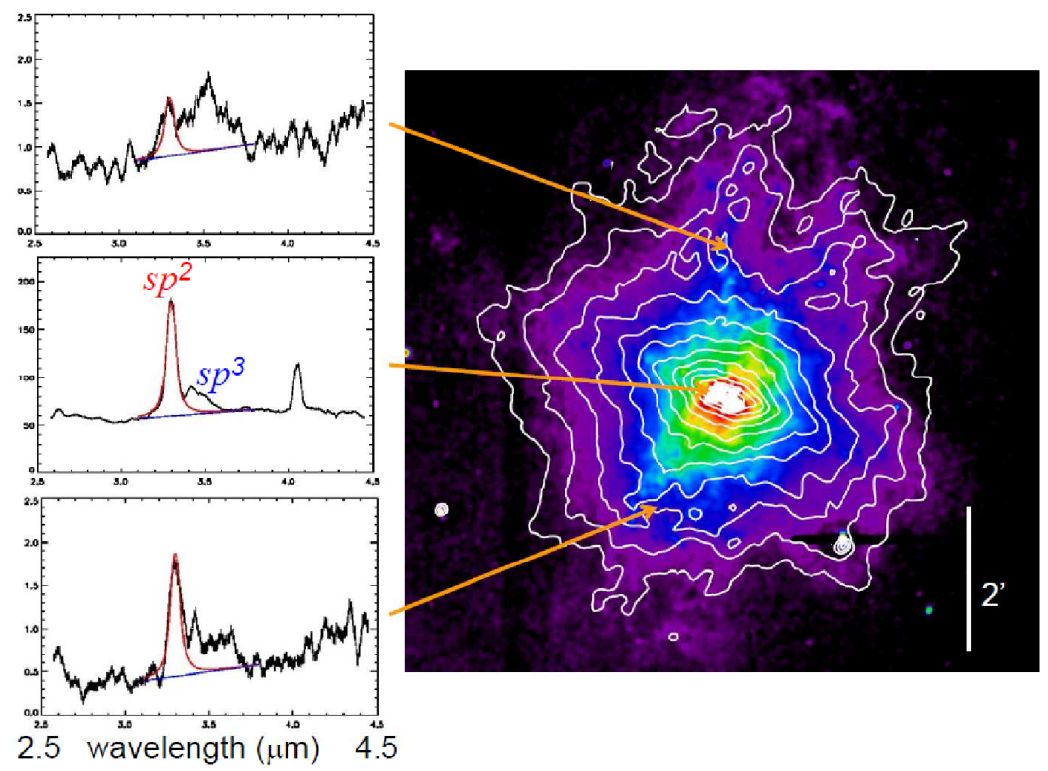

Figure 3: Contour map of M 82 in the AKARI $7 \mu \mathrm{m}$ (PAH) band overlaid on the H $\alpha$ images [51]. The 2.5-4.5 $\mu \mathrm{m}$ spectra obtained by the AKARI near-IR spectroscopy are shown together, which are taken from the positions indicated in the $7 \mu \mathrm{m}$ map.

considerably decreases toward the luminous end in the ULIRG population. Local ULIRGs may intrinsically possess smaller amounts of PAHs relative to BGs, as a result of PAH processing through recent galaxy mergers. Some fraction of PAHs may have been destroyed once by shocks which occurred during a merging process, whereas BGs survive, which is consistent with the observational indication that local ULIRGs are merging galaxies (e.g. [59]).

\section{Properties of dust in passive galaxies}

Elliptical galaxies provide us with another extreme case, representing the end of the lifecycle of dust. In such passive galaxies, the origin of dust itself has been a matter of debate (e.g. 㺻). Some elliptical galaxies contain surprisingly large dust masses, considering the efficient destruction by sputtering in the hot plasma environments [60, 61, 62]. Spitzer observed many early-type galaxies, but did not clearly show a significant correlation between their far-IR luminosities and $B$-band luminosities [63]. This result suggests that the amount of the BGs remaining in the galaxies may not be related to the stellar mass loss of evolved cool stars. Hence a clear conclusion on whether a majority of dust is of internal (stellar) origin or external (gas-rich merger) origin is yet to be reached. As described above, Spitzer detected PAH emission in elliptical galaxies with unusual band ratios; the usually strongest $7.7 \mu \mathrm{m}$ feature is notably weak, whereas the $11.3 \mu \mathrm{m}$ feature is relatively strong. It is concluded that neutral PAHs, rather than ionized ones, become dominant in very soft radiation fields, typical of elliptical galaxies, giving rise to the faint $\mathrm{C}-\mathrm{C}$ vibration features at 6-8 $\mu \mathrm{m}$. It should be noted that the PAH $11.3 \mu \mathrm{m}$ emission does not represent any star-forming activity in this case, although PAH emission is often used as star-formation indicators for studies of galaxies. It is suggested that the AGN-assisted feedback outflow from a central reservoir may play 

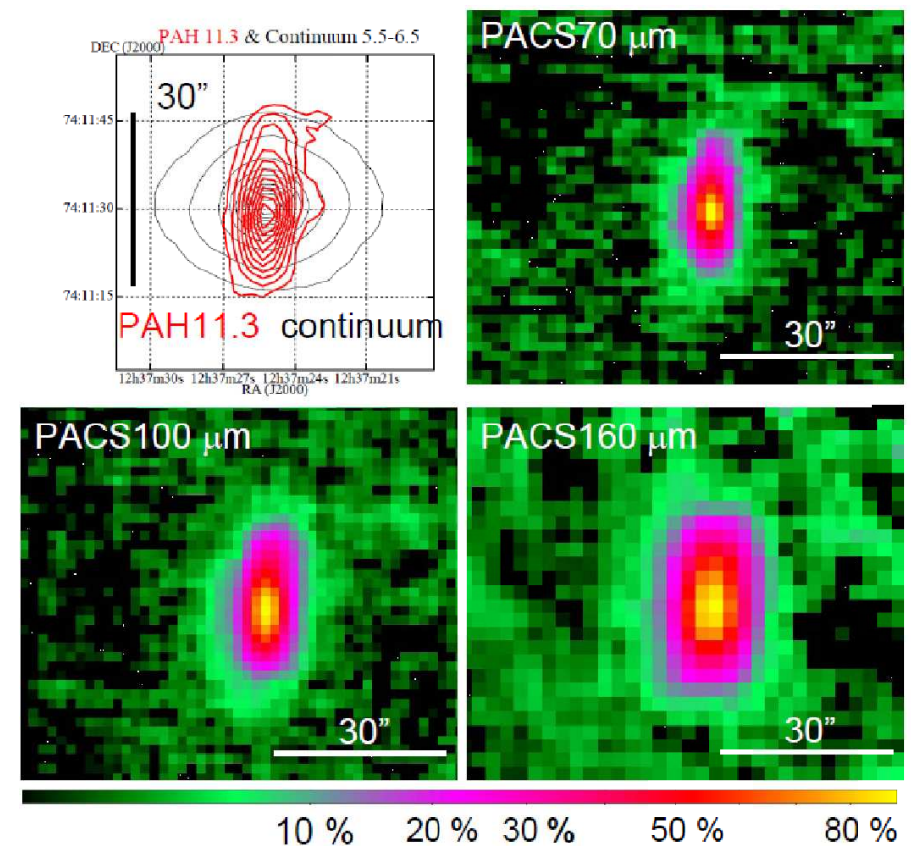

Figure 4: Spitzer/IRS spectral mapping images of the PAH $11.3 \mu \mathrm{m}$ feature and 5.5-6.5 $\mu \mathrm{m}$ continuum emission for the elliptical galaxy NGC 4589 [66]. The Herschel/PACS 70, 100 and $160 \mu \mathrm{m}$ maps of the same galaxies.

an important role in supplying dust into the interstellar space of elliptical galaxies [64, 65]; PAHs might come from residual dust fragments originating from the sputtering of large grains.

Figure 7 shows the spatial distribution of PAHs in an elliptical galaxy, NGC 4589, together with that of the stellar $(5.5-6.5 \mu \mathrm{m})$ continuum emission [66]. The distributions of both the PAH $11.3 \mu \mathrm{m}$ and the continuum emission were obtained with Spitzer/IRS spectral mapping observations. The figure reveals that the PAHs are compactly distributed along a minor-axis dust lane in the galactic center. Figure 7 also shows the spatial distribution of dust in NGC 4589 at 70, 100 and $160 \mu \mathrm{m}$, obtained by deep imaging observations with Herschel/PACS (Kaneda et al. in prep.). The far-IR dust emission is spatially well resolved, exhibiting distributions quite similar to PAHs. Since PAHs are likely to be old remnants originating from mass losses from intermediate-mass stars, while silicate grains are currently being produced by low-mass evolved stars (if they are both of internal origin), this similarity may have deep physical implications for the evolution of the ISM in old galaxies. The AKARI near-IR spectroscopy of NGC 4589 shows strong absorption features due to $\mathrm{SiO}$ and $\mathrm{CO}$ around $4.2-4.7 \mu \mathrm{m}$ from much wider areas of the galaxy than the PAH and dust emission [67]. These absorption features are most likely to originate from low-mass O-rich stars [68], which are currently supplying silicate but not carbonaceous grains. Hence the Herschel observation suggests that the silicate grains being currently produced by low-mass O-rich stars make only a minor contribution to the total far-IR dust emission observed in NGC 4589. 


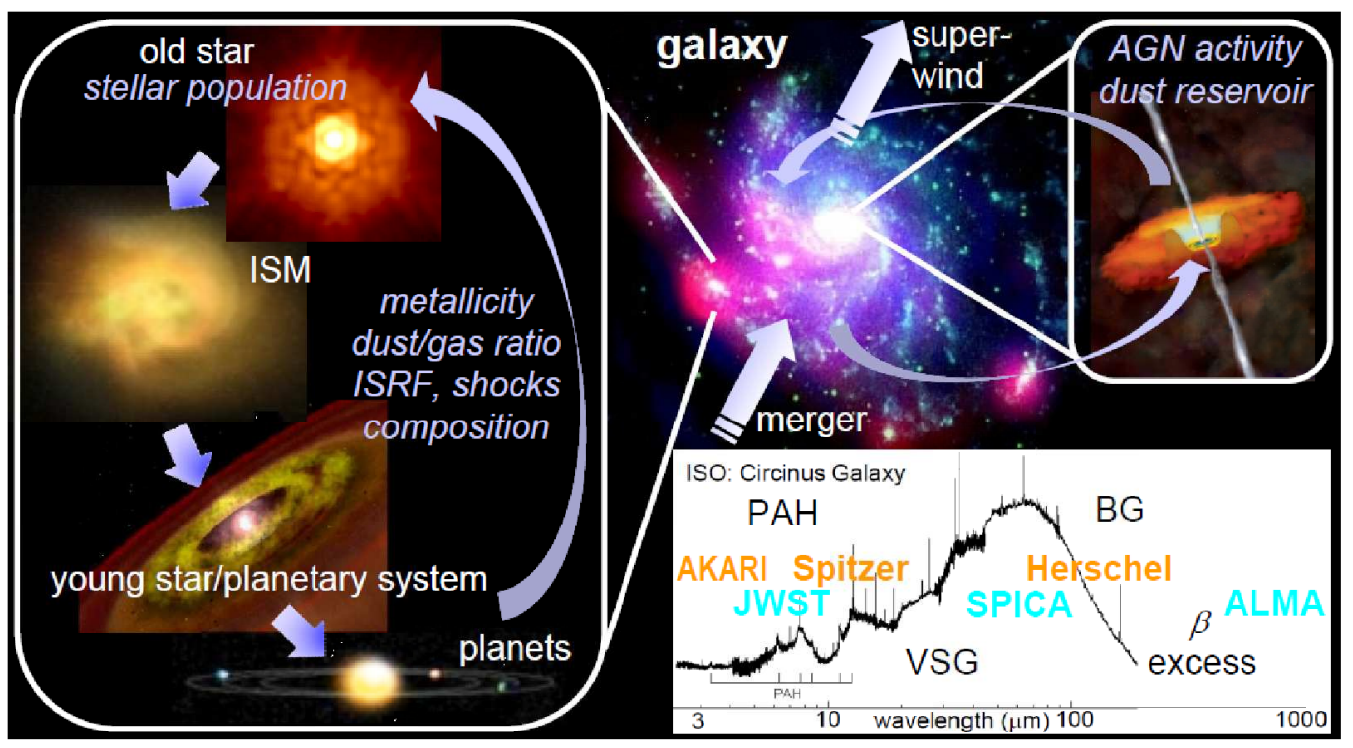

Figure 5: Illustration to show that nearby galaxies are important to unravel the whole story of the life cycle of dust in the universe with the current and future major observational facilities.

\section{Summary and future}

Thanks to the development of IR observational technologies in space, structures of nearby galaxies are now spatially resolved in PAH, VSG and BG emission, which has enabled us to discuss their relationships in some detail. In particular, the understanding of the PAH emission in galaxies has improved very much with the advent of Spitzer, while many pieces of information on the distributions of BGs within galaxies and their spatially-resolved properties has been obtained with Herschel. The AKARI all-sky surveys and near-IR spectroscopy will complement the Spitzer and Herschel studies of nearby galaxies. Among the results presented above, the mass fraction of PAHs to BGs and the PAH band ratios are found to vary substantially with the interstellar environments in galaxies: for example, with the intense radiation field in dwarf galaxies, giant HII regions in spiral galaxies, and the soft radiation field in early-type galaxies. The behavior of the dust opacity in the far-IR to sub-mm region including the $500 \mu \mathrm{m}$ excess and the aliphatic to aromatic ratios in the near-IR are also useful probes of the interstellar environment, especially on the degree of dust processing in mildly shocked regions. These two results are important to understand the fate of dust processed by shocks and radiation through the evolution of galaxies. The chemical compositions of VSGs and BGs are, however, still quite unknown observationally, and future mid- to far-IR spectral data which fills the gap between Spitzer and Herschel (Fig. 5) would be crucial to understand their compositions.

\section{References}

[1] G. Neugebauer, G. K. Miley, B. T. Soifer and P. E. Clegg, Quasars measured by the Infrared Astronomical Satellite, 1986, ApJ 308815. 
[2] D. B. Sanders, E. S. Phinney, G. Neugebauer, B. T. Soifer, and K. Matthews, Continuum energy distribution of quasars - Shapes and origins, 1989, ApJ 34729.

[3] M. H. K. de Grijp, G. K. Miley, J. Lub, and T. G. de Jong, Infrared Seyferts - A new population of active galaxies?, 1985, Nature $\mathbf{3 1 4} 240$.

[4] G. R. Knapp, P. Guhathakurta, D.-W. Kim, and M. A. Jura, Interstellar matter in early-type galaxies. I - IRAS flux densities, 1989, ApJS 70329.

[5] B. T. Soifer, et al., Infrared galaxies in the IRAS minisurvey, 1984, ApJ 278 L71.

[6] D. B. Sanders and I. F. Mirabel, Luminous Infrared Galaxies, 1996, ARA\&A 34749.

[7] A. F. M. Moorwood, ISO observations of active galaxies, 1999, ESA-SP 427825.

[8] E. Sturm, et al., ISO-SWS spectra of galaxies: Continuum and features, 2000, A\&A 358481.

[9] N. Lu, et al., Infrared Emission of Normal Galaxies from 2.5 to 12 Micron: Infrared Space Observatory Spectra, Near-Infrared Continuum, and Mid-Infrared Emission Features, 2003, ApJ 588 199.

[10] R. C. Kennicutt, et al., SINGS: The SIRTF Nearby Galaxies Survey, 2003, PASP 115928.

[11] H. Kaneda, B.-C. Koo, T. Onaka, and H. Takahashi, AKARI observations of the ISM in our Galaxy and nearby galaxies, 2009, AdSpR 441038.

[12] R. C. Kennicutt, et al., KINGFISH - Key Insights on Nearby Galaxies: A Far-Infrared Survey with Herschel: Survey Description and Image Atlas, 2011, PASP 1231347.

[13] S. C. Madden, et al., An Overview of the Dwarf Galaxy Survey, 2013, PASP 125600.

[14] J. D. T. Smith, et al., The Mid-Infrared Spectrum of Star-forming Galaxies: Global Properties of Polycyclic Aromatic Hydrocarbon Emission, 2007, ApJ 656770.

[15] E. Sturm, et al., Mid-Infrared Diagnostics of LINERS, 2006, ApJ 65313.

[16] C. Leipski, R. Antonucci, P. Ogle, and D. Whysong, The Spitzer View of FR I Radio Galaxies: On the Origin of the Nuclear Mid-Infrared Continuum, 2009, ApJ 701891.

[17] H. Kaneda, T. Onaka, and I. Sakon, Detection of PAH Emission Features from Nearby Elliptical Galaxies with the Spitzer Infrared Spectrograph, 2005, ApJ 632 L83.

[18] H. Kaneda, T. Onaka, I. Sakon, T. Kitayama, Y. Okada, and T. Suzuki, Properties of Polycyclic Aromatic Hydrocarbons in Local Elliptical Galaxies Revealed by the Infrared Spectrograph on Spitzer, 2008, ApJ $\mathbf{6 8 4} 270$.

[19] P. Panuzzo, et al., Nearby early-type galaxies with ionized gas. VI. The Spitzer-IRS view. Basic data set analysis and empirical spectral classification, 2011, A\&A $\mathbf{5 2 8} 10$.

[20] R. Rampazzo, P. Panuzzo, O. Vega, A. Marino, A. Bressan, and M. S. Clemens, A Spitzer-IRS spectroscopic atlas of early-type galaxies in the Revised Shapley-Ames Catalog, 2013, MNRAS 432 374.

[21] B. T. Draine and A. Li, Infrared Emission from Interstellar Dust. IV. The Silicate-Graphite-PAH Model in the Post-Spitzer Era, 2007, ApJ 657810.

[22] G. J. Bendo, et al., The relations among 8, 24 and $160 \mu \mathrm{m}$ dust emission within nearby spiral galaxies, 2008, MNRAS 389629. 
[23] C. W. Engelbracht, K. D. Gordon, G. H. Rieke, M. W. Werner, D. A. Dale, and W. B. Latter, Metallicity Effects on Mid-Infrared Colors and the $8 \mu \mathrm{m}$ PAH Emission in Galaxies, 2005, ApJ 628 L29.

[24] C. W. Engelbracht, et al., Metallicity Effects on Dust Properties in Starbursting Galaxies, 2008, ApJ 678804.

[25] M. Galametz, et al., Mapping the cold dust temperatures and masses of nearby KINGFISH galaxies with Herschel, 2012, MNRAS 425763.

[26] A. Rémy-Ruyer, et al., Revealing the cold dust in low-metallicity environments. I. Photometry analysis of the Dwarf Galaxy Survey with Herschel, 1985, A\&A 55795.

[27] C. Meny, V. Gromov, N. Boudet, J.-Ph. Bernard, D. Paradis, and C. Nayral, Far-infrared to millimeter astrophysical dust emission. I. A model based on physical properties of amorphous solids, 2007, A\&A 468171.

[28] B. T. Draine and B. Hensley, Magnetic Nanoparticles in the Interstellar Medium: Emission Spectrum and Polarization, 2013, ApJ 765159.

[29] A. Kirkpatrick, et al., Investigating the Presence of 500 Eìm Submillimeter Excess Emission in Local Star Forming Galaxies, 2013, ApJ 77851.

[30] F.-X. Désert, F. Boulanger, and J. L. Puget, Interstellar dust models for extinction and emission, 1990, A\&A 237215.

[31] B. T. Draine and H.-M. Lee, Optical properties of interstellar graphite and silicate grains, 1984, ApJ 28589.

[32] M. Compiègne, et al., The global dust SED: tracing the nature and evolution of dust with DustEM, $2011, A \& A \mathbf{5 2 5} 103$.

[33] V. G. Zubko, V. Mennella, L. Colangeli, and E. Bussoletti, Optical constants of cosmic carbon analogue grains - I. Simulation of clustering by a modified continuous distribution of ellipsoids, 1996, MNRAS 2821321.

[34] J. S. Mathis, P. G. Mezger, and N. Panagia, Interstellar radiation field and dust temperatures in the diffuse interstellar matter and in giant molecular clouds, 1983, A\&A 128212.

[35] D. A. Dale and G. Helou, The Infrared Spectral Energy Distribution of Normal Star-forming Galaxies: Calibration at Far-Infrared and Submillimeter Wavelengths, 2002, ApJ 576159.

[36] F. Galliano, S. C. Madden, A. P. Jones, C. D. Wilson, J.-P. Bernard, and F. Le Peintre, ISM properties in low-metallicity environments. II. The dust spectral energy distribution of NGC 1569, 2003, A\&A 407159.

[37] F. Galliano, S. C. Madden, A. P. Jones, C. D. Wilson, and J.-P. Bernard, ISM properties in low-metallicity environments. III. The spectral energy distributions of II $\mathrm{Zw} 40$, He 2-10 and NGC 1140, 2005, A\&A 434867.

[38] B. T. Draine, et al., Dust Masses, PAH Abundances, and Starlight Intensities in the SINGS Galaxy Sample, 2007, ApJ 663866.

[39] D. A. Dale, et al., Herschel Far-infrared and Submillimeter Photometry for the KINGFISH Sample of nearby Galaxies, 2012, ApJ 74595.

[40] G. Aniano, et al., Modeling Dust and Starlight in Galaxies Observed by Spitzer and Herschel: NGC 628 and NGC 6946, 2012, ApJ 756138. 
[41] M. S. Khramtsova, D. S. Wiebe, P. A. Boley, and Ya. N. Pavlyuchenkov, Polycyclic aromatic hydrocarbons in spatially resolved extragalactic star-forming complexes, 2013, MNRAS 4312006.

[42] K. M. Sandstrom, A. D. Bolatto, B. T. Draine, C. Bot, and S. Stanimirović, The Spitzer Survey of the Small Magellanic Cloud $\left(S^{3} M C\right)$ : Insights into the Life Cycle of Polycyclic Aromatic Hydrocarbons, 2010, ApJ 715701.

[43] M. Galametz, et al., Herschel photometric observations of the nearby low metallicity irregular galaxy NGC 6822, 2010, A\&A $\mathbf{5 1 8} 55$.

[44] P. C. Frisch, et al., Dust in the Local Interstellar Wind, 2013, ApJ 525492.

[45] A. G. G. M. Tielens, C. F. McKee, C. G. Seab, and D. J. Hollenbach, The physics of grain-grain collisions and gas-grain sputtering in interstellar shocks, 1994, ApJ 431321.

[46] A. P. Jones, A. G. G. M. Tielens, and D. J. Hollenbach, Grain Shattering in Shocks: The Interstellar Grain Size Distribution, 1996, ApJ 469740.

[47] D. Ishihara, H. Kaneda, T. Onaka, Y. Ita, M. Matsuura, and N. Matsunaga, Galactic distributions of carbon- and oxygen-rich AGB stars revealed by the AKARI mid-infrared all-sky survey, 2011, A\&A 53479.

[48] E. Schlömann, Dielectric Losses in Ionic Crystals with Disordered Charge Distributions, 1964, Phys.Rev.A 135413.

[49] N. I. Agladze, A. J. Sievers, S. A. Jones, J. M. Burlitch, and S. V. W. Beckwith, S. V. W., Reassessment of millimetre-wave absorption coefficients in interstellar silicate grains, 1994, Nature 372243.

[50] C. W. Engelbracht, et al., Extended Mid-Infrared Aromatic Feature Emission in M82, 2006, ApJ 642 L127.

[51] H. Kaneda, et al., Large-scale distributions of mid-and far-infrared emission from the center to the halo of $M 82$ revealed with AKARI, 2010, A\&A 514 A14.

[52] H. Roussel, SPIRE imaging of M 82: Cool dust in the wind and tidal streams, 2010, A\&A 518 L66.

[53] G. Thuma, N. Neininger, U. Klein, and R. Wielebinski, Cold dust in the starburst galaxy M 82, 2000, $A \& A 35865$.

[54] M. Yoshida, K. S. Kawabata, and Y. Ohyama, Spectropolarimetry of the Superwind Filaments of the Starburst Galaxy M 82: Kinematics of Dust Outflow, 2011, PASJ 63493.

[55] A. P. Jones, L. Fanciullo, M. Köhler, L. Verstraete, V. Guillet, M. Bocchio, and N. Ysard, The evolution of amorphous hydrocarbons in the ISM: dust modelling from a new vantage point, 2013, $A \& A 55862$.

[56] M. Yamagishi, et al., AKARI near-infrared spectroscopy of the aromatic and aliphatic hydrocarbon emission features in the galactic superwind of M 82, 2012, A\&A 541 A10.

[57] R. Yamada, S. Oyabu, H. Kaneda, M. Yamagishi, D. Ishihara, J.-H. Kim, and M. Im, A Relation of the PAH $3.3 \mu \mathrm{m}$ Feature with Star-forming Activity for Galaxies with a Wide Range of Infrared Luminosity, 2013, PASJ 65103.

[58] H. Mouri, K. Kawara, Y. Takeushi, and M. Nishida, A relation between H2 V=1-0 S(1) and 3.28 micron emission in Seyfert and starburst galaxies, 1990, ApJ 356 L39.

[59] D. L. Clements, W. J. Sutherland, R. G. McMahon, and W. Saunders, Optical imaging of ultraluminous IRAS galaxies: how many are mergers?, 1996, MNRAS 279477. 
[60] P. Goudfrooij and T. de Jong, Interstellar matter in Shapley-Ames elliptical galaxies. IV. A diffusely distributed component of dust and its effect on colour gradients, 1995, A\&A 298784.

[61] P. Temi, F. Brighenti, W. G. Mathews, and J. D. Bregman, Cold Dust in Early-Type Galaxies. I. Observations, 2004, ApJS 151237.

[62] H. Kaneda, T. Onaka, T. Kitayama, Y. Okada, and I. Sakon, Dust in Hot Plasma of Nearby Dusty Elliptical Galaxies Observed with the Spitzer Space Telescope, 2007, PASJ 59107.

[63] P. Temi, F. Brighenti, and W. G. Mathews, Far-Infrared Spitzer Observations of Elliptical Galaxies: Evidence for Extended Diffuse Dust, 2007, ApJ 6601215.

[64] P. Temi, F. Brighenti, and W. G. Mathews, Spitzer Observations of Transient, Extended Dust in Two Elliptical Galaxies: New Evidence of Recent Feedback Energy Release in Galactic Cores, 2007, ApJ 666222.

[65] W. G. Mathews, P. Temi, F. Brighenti, and A. Alexandre, Variations of Mid-and Far-infrared Luminosities among Early-type Galaxies: Relation to Stellar Metallicity and Cold Dust, 2013, ApJ 76828.

[66] H. Kaneda, et al., Polycyclic Aromatic Hydrocarbon Emission from the Dust Lane of an Elliptical Galaxy with the Spitzer Infrared Spectrograph, 2010, ApJ 716 L161.

[67] T. Mori, S. Oyabu, H. Kaneda, D. Ishihara, and M. Yamagishi, Properties of Dust in Early-Type Galaxies Based on the All-Sky Data and Near-Infrared Spectra, 2012, PKAS 27263.

[68] A. M. Heras, et al., Infrared spectral classification of normal stars, 2013, A\&A 394539. 